\title{
Functional connectivity patterns of ERPs activity during the generation of global and local imagery Danni Sui ${ }^{1}$, Qingbai Zhao ${ }^{1}$ and Yiyuan Tang*1,2
}

\author{
Address: ${ }^{1}$ Institute of Neuroinformatics and Laboratory for Brain and Mind, Dalian University of Technology, Dalian 116024, PR China and \\ ${ }^{2}$ Department of Psychology, University of Oregon, Eugene, OR 97403, USA \\ Email: Yiyuan Tang* - yiyuan@uoregon.edu \\ * Corresponding author
}

from Eighteenth Annual Computational Neuroscience Meeting: CNS*2009

Berlin, Germany. 18-23 July 2009

Published: 13 July 2009

BMC Neuroscience 2009, I0(Suppl I):P7| doi:I0.I|86/I47|-2202-I0-SI-P7|

This abstract is available from: http://www.biomedcentral.com/I47I-2202/I0/SI/P7 I

(c) 2009 Sui et al; licensee BioMed Central Ltd.

\section{Introduction}

Global and local visual processing are well established, but the literature on global and local imagery processing is sparse. To analyze the organization of functional connectivity of event-related potentials (ERPs) activity during the generation of global and local imagery, we evaluated the spatial patterns of ERPs correlation between all possible pairs of electrodes (60) placed over the scalp of 28 healthy young normal subjects participating in a global/ local imagery generation task using two graph theoretical measures: the clustering coefficient and average path length [1-3].

\section{Results and discussion}

Results showed that the functional network of global imagery generation produced a shorter average path length, while that of local imagery generation got a larger clustering coefficient. Furthermore, the average distance between functional connected electrodes was longer in the generation of global imagery than in local imagery. We suggested that the difference of functional connectivity patterns might reflect the different processing modes of the generation of global and local imagery that global imagery emphasized particularly on global integration, while local imagery on local specialization.

\section{Acknowledgements}

This work was supported by NSFC 30670699 , NCET-06-0277 and the

James S. Bower Foundation.

\section{References}

I. Doricchi F, Armati C, Martorano G, Violani C: Generation of skeletal and multipart mental visual images in the cerebral hemispheres: A study in normal subjects. Neuropsychologia 1994, 33:8I-20I.

2. Sui DN, Tang YY, Yu QB, Wang JH, Wang W: The generation of global and local mental imagery and its Gender Differences. Prog Mod Biomed 2007, 7:1554-1556.

3. Watts DJ, Strogatz SH: Collective dynamics of 'small-world' networks. Nature 1998, 393:440-442. 